\title{
EVOLUTION OF X-RAY CLUSTERS OF GALAXIES WITH
} ACTIVE PROTOGALAXIES

\author{
H. SAGA, S. YACHI AND A. HABE \\ Graduate School of Science, Hokkaido University, Sapporo, JAPAN
}

\section{Introduction}

We consider heating due to proto galaxies in the formation process of clusters of galaxies, since much metal is observed in the intracluster gas which must be ejected from protogalaxies vir strong galactic winds and the metal abundance in the intracluster gas correlates with the fraction of early type galaxies in clusters (Arnaud 1993). We also consider radiative cooling. From the difference between the observed $L_{X}-T_{X}$ relation of X-ray cluster (Hatsukade 1989), $L_{X} \propto T_{X}^{2.7-3.3}$, and the prediction from the self-similar model (Kaiser 1986), $L_{X} \propto T_{X}^{2}$, it is pointed out that physical processes which are not taken into account in the self-similar model, e.g., effect of radiative cooling, and/or effect of proto galaxy heating, play a important role in the formation process (Evrard and Henry 1991, Kaiser 1991).

\section{Model and Numerical Results}

We assume that the galaxy formation begins from $z=3$ for $10^{8.5} \mathrm{yr}$ at density maxima selected by the friend-friend algorithm at $\mathrm{z}=3$. During the galaxy formation, supernovae explode and add metal and heat energy to gas in these regions. Our numerical calculation code is N-body code by using the GRAPE3A for dark matter and SPH code for gas motion. Numbers of both particles are 50000 .

From our numerical results of the non-heating models, power index of the $L_{X}-T_{X}$ relation is 2.3 , which is larger than the self-similar model prediction. This power index value is well agree with the numerical results by Kang et al. (1994). The power index value of the non-heating models is larger than the power index value of the self-similar model. From the 
heating models of $\epsilon=0.4$ the power index of $L_{X}-T_{X}$ is 2.6, which is larger than the non-heating model results, and are close to the observed power index of the $L_{X}-T_{X}$ relation, where $\epsilon=0.4$ correspond to heating due to about half enegy released by supernova in protogalaxies if the initial mass function is the Salpeter' one. Considering radiative cooling in the heating model, the power index of $L_{X}-T_{X}$ is 2.2 ,

\section{Discussion}

If we assign metal abundance of intracluster gas, we can estimate the formation efficiency of galaxies and the heating efficiency from our numerical results.

From our numerical results, we suggest that (1) high z X-ray luminous clusters are metal rich, and (2) small clusters are metal abundant. These properties are consistent with GINGA data (Arnaud 1990), although ASCA data is different from it (Ohashi 1995).

Radiative cooling reduces the effect of heating by protogalaxies, especially for small clusters, since small clusters have low temperature of gas. As a result, the $L_{x}-T_{x}$ relation becomes similar to the non-heating case. Additional heating, such as heating due to type I supernovae after initial star burst, can make a steep $L_{x}-T_{x}$ relation.

\section{references}

Arnaud,M, 1994, in Cosmological Aspects of X-ray clusters of galaxies, ed. by W,C,Seitter, Kluwer Academic Publishers, p 197.

Evrard,A.E. and Henry J.P., 1991, Ap.J. 383, 95.

Hatsukade I, 1989, Phd. thesis in ISAS research note 435.

Kaiser,N. 1986, M.N., 222, 323.

Kaiser,N. 1991, Ap.J., 383, 104.

Kang, H. et al., 1994, Ap.J., 430, 83.

Ohashi, T., 1995, private communication 